\title{
COLOR IN PLATINUM FILMS.
}

\author{
By ISABELLE STONE.
}

WHILE making platinum films by cathode discharge I found that, using a platinum point as cathode, I obtained colored rings on a glass plate placed directly under the point. In order to investigate this phenomenon, I got together the necessary parts for a receiver of the kind shown in the diagram. The receiver $A$ fitted into a circular groove cut out of the aluminum plate $x y$. This aluminum plate contained a binding post $B$, and inside the receiver, two aluminum supports $K$ and $L$ on which the glass plate $J$ rested. Through the neck of the receiver fitted a hollow ground-glass stopper $C$. $C$ was in connection with two branch tubes $D$ and $E$ in each of which fitted ground-glass stopcocks $F$ and $G$. Through the top of the ground-glass stopper $C$ was fused a loop of platinum wire ending

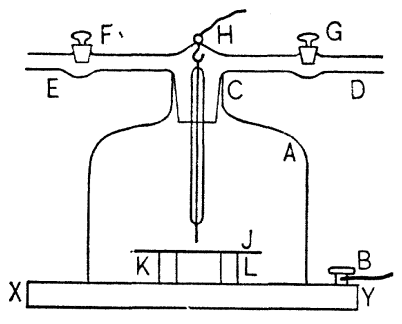

Fig. 1. within the receiver in a hook. To the outer loop $H$ the wire from the secondary coil was attached. To the inner hook any form of cathode could be suspended. The cathode first used was a thick platinum wire sealed into a glass tube with enough of the wire left at the upper end to form a loop for suspension and two millimeters left at the lower end to form the cathode. The stopcock $G$ was turned to shut off the entrance of air and the tube $E$ being joined through a drying tube to a pulsometer oil pump, stopcock $F$ was left open and the exhaustion of air from the receiver commenced. Later, when it became necessary to measure accurately the pressure of the air in the receiver, the tube $D$ was connected to a McLeod gauge by means of the two-way stopcock $G$. The pulsometer oil pump was run by a motor and made about thirty strokes per min- 
ute and by means of it I could get down to a pressure of $.3 \mathrm{~mm}$. A wax of rosin and beeswax was used to make the connection between the receiver and the aluminum base. The first fourteen plates were made with no account taken of pressure except that a purple light in the receiver meant a low vacuum and the pale blue light, high vacuum; direct current was used, the alternations brought about by a Wehnelt interrupter and secondary coil. This interrupter was first a thin platinum wire immersed in dilute sulphuric acid; the wire was surrounded by a hard-rubber cylinder except for its tip which dipped in the acid and could be lengthened as it wore away. It was an unsatisfactory interrupter for continuous work, since it could be run for only a minute or two at a time, then would sputter and cease working. However, if rested for a moment and thus allowed to cool, it would again work satisfactorily for a minute or two; thus a summation of effects could be obtained. Later, a solid stick of platinum was substituted for the fine wire and could be used consecutively for an hour or more at a time and in this respect was all that could be desired. But even with the platinum stick interrupter, the current density would change as the point wore away and could not be measured with any accuracy, therefore, in the end the Wehnelt interrupter and coil were given up entirely and a 2,000-volt direct-current dynamo used whose current could be accurately measured at any time. Another improvement consisted in connecting the receiver directly to a mercury pump so that lower pressures could easily be obtained and by connecting the oil pump to the mercury pump, the air could be removed from the receiver quickly and with ease - the pressure was read from a McLeod gauge connected to the mercury pump.

Viewed through the receiver, the glass plate could be watched during the course of the deposit and the general method of formation appeared as follows: A brown spot formed directly under the cathode; this changed gradually into reddish brown, increasing in size; then blue appeared at the center edged with red and beyond the red the brown, later blue increased in area and turned to red, indicating the starting of the second set of colored rings.

It soon became evident that time of discharge and distance between cathode point and glass plate were closely related; the closer the 
point to the plate, the shorter the time taken to get good colors and conversely. I also found that with the plate close to, say within $2 \mathrm{~mm}$. of the cathode, the rings were narrow and of small diameter. As the distance was increased the rings spread out in area, became broader and of larger diameter. By varying the distance from 2 to $10 \mathrm{~mm}$., the outside diameter of the rings could be increased from a few millimeters to several centimeters. This was true, whatever the form of cathode used. Also, on changing my cathode from coarse to fine wire, the rings became narrower and of smaller diameter.

I soon found that pressure played an important part in the formation of the rings and, after the first few films, kept a strict account of that factor.

In my early work I obtained many films that at the time of making could not be accounted for, but showed typical aspects after finding the important part that pressure and distance played. I frequently obtained dim rings, now and then a granular deposit instead of the smooth coating generally gotten and a particular kind of film showing a transparent center surrounded by a black irregular ring often puzzled me, for I was soon led to see that good colored rings had two characteristic appearances. One type showed a faint yellow tinge at the center, the other colored rings were practically transparent and beyond the colors was a wide absolutely transparent zone bounded by a fine black deposit. The other type of good colored rings showed brilliant colors and were absolutely transparent. To account for these two types and to explain variations from them, it was necessary to make many films under varying and measurable conditions.

After the cathode had been used for a time, a heavy platinum coating would collect on the glass surrounding the platinum tip. As this increased, the cathode glow would bend up about the glass, away from the tip. When this occurred, it was hopeless to accomplish anything without great waste of time, for the discharge was made very slowly and most irregularly and the best thing to do was to stop and clean off the glass.

New forms of cathodes were tried. A platinum sphere fused on the end of the heavy platinum wire gave rings like those produced 
by the platinum point. By varying the distance between cathode and plate, the same peculiarities in size of ring was noted as with the point cathode. I tried a cathode made of thin platinum wire bent into a circle with the plane of the circle horizontally above the glass plate. The point of joining of the circle to the supporting stem always produced a little distortion of the inner rings ; but, in general, under the circular opening of the cathode there was a uniform area of color; the rings were under the wire and followed in outline the bending of the wire; the deposit directly under the wire was thickest, showing a brownish color. In any vertical section of such a cathode, different points of the wire are at different distances from the plate and in consequence the rings farther from the wire are broader than those more directly under the wire. A lack of parallelism between the circle cathode and the plate was indicated by a crescent that occurred in the rings. I gradually bent the circular cathode down until it stood vertically over the plate. Plates were made with the circle at various angles with the vertical. These plates showed the following characteristic; about that part of the wire nearest the plate, the rings were narrowest, taking an elliptical form, the lack of symmetry being on that side where the circle was farthest from the plate. This distortion decreased as the angle made with the vertical was decreased, until when the cathode stood with its plane perpendicular to the plate, all the ellipses were symmetrical. In this series of experiments, there existed in the intermediate stages what might be called a total effect and an effect due to that part of the cathode nearest the plate - the total effect being shown by the bounding elliptical rings and the special effect in the distorted and eccentric ellipses within. When the circular cathode hung perpendicularly to the plate, the special effect merged into the total effect and the plate was covered with perfectly symmetrical ellipses. After observing that an elliptical cathode gave ellipses and a triangular cathode, triangles, I tried for side effects given by the different cathodes standing my plate vertically in the receiver and near to that portion of the cathode to be investigated. Bending my circular cathode, so that its plane stood vertically and also in such a way that the effect of the supporting stem was eliminated, I obtained on a vertical plate, parallel to the cathode plane, circular 
rings and on a vertical plate at right angles to the cathode plane, elliptical rings as would be expected. With the elliptical cathode, ellipses were formed whether the vertical plate was placed opposite the side of least or greatest curvature, though, while in the first case, the ellipses were long and narrow, in the second case, they were almost circular; the side effects for the knob cathode were always circles. With a cathode shaped, thus,

and a horizontal plate, I obtained long, narrow ellipses, the length of the ellipse depending on the length of the wire and the width of the ellipse on the diameter of the wire; a plate placed vertically at the left hand end received ellipses and at the right hand end, namely the point, circles were obtained. The same kind of ellipses were obtained from a circular cathode hung horizontally and a vertical plate, no matter where the plate was placed, as in this case the curvature is everywhere the same.

Any change in form of cathode produced a corresponding change in form of rings and in each case, the deposit under that part of the cathode nearest the plate showed a yellow hue; beyond the colored rings was a transparent zone following the outline of the rings and beyond this zone an even black deposit. As previously noted in the case of the circular cathode, it was found that in all cases where the wire was bent into various shapes, a lack of parallelism between the plane of the cathode and the plate was indicated by a crescent of color among the symmetrical rings. Other sources of distortion of rings occurred when the discharge passed to the aluminum supports, when the plate was too small and did not cover the supports, and also when there were irregularities on the cathode. Blemishes sometimes appeared due to testing the discharge at high pressure, especially when the current was strong. In bringing the plate close to the cathode, particularly in obtaining side effects, a portion of the cathode would touch the plate and even if at first the cathode was not touching, it was noticed that as soon as the discharge passed, the cathode would be attracted to the glass and touch it througout the interval of film formation. This touching could always be traced by a transparency of the plate where the contact was, every other part of the plate having a deposit. 
From experience with trying to get a half film of platinum on an interferometer plate, working twenty-four hours with the lowest pressure the oil pump would give and getting rectangular rings on the plate (like the shape of the rectangular cathode used), I realized the importance of pressure, so put my receiver on a mercury air pump with which I could obtain pressures of any value desired. I wished to find whether a low pressure produced a uniform field and no color as Mr. Langden had found. ${ }^{1}$ With a knob-cathode at a very short distance from the plate and a pressure of about O. I $\mathrm{mm}$. I obtained a transparent center surrounded by a black ring and no color. On using a pressure of $1.28 \mathrm{~mm}$. and the same distance $(2$ $\mathrm{mm}$.) I obtained in three minutes beautiful colored rings, showing the brown tint characteristic previously noted. Taking a given distance, I tried the effect of altering the pressure, using $1.28 \mathrm{~mm}$., 0.9 $\mathrm{mm}$., $0.6 \mathrm{~mm}$., $0.3 \mathrm{~mm}$. and $0.1 \mathrm{~mm}$. pressures. I obtained colors until $0.3 \mathrm{~mm}$. pressure was reached at which there was a heavy deposit, showing a trace of ring formation on the back of the plate. At $\mathrm{O}$. I mm. pressure, there was but a slight deposit, center transparent, no color. I then tried altering the distance but taking the same pressures used before. All the charateristics previously noted were obtained. At 0.3 and $0.1 \mathrm{~mm}$. pressure no colors were found but heavier deposits than for the shorter distance. Thus, when the Wehnelt interrupter was used, the minimum pressure for rings seemed to be about $0.3 \mathrm{~mm}$. Then I tried to find the maximum pressure for ring formation taking a fixed distance and varying the pressure from $0.1 \mathrm{~mm}$. as high as I could go and still get color. At $3 \mathrm{~mm}$. pressure there was considerable trace of color, though the deposit was irregular. For good ring formation $2 \mathrm{~mm}$. seemed the maximum pressure when the Wehnelt was used. So I could state that using this interrupter the minimum pressure for rings was $0.3 \mathrm{~mm}$., and the maximum pressure about $2 \mathrm{~mm}$. On using the 2,000-volt dynamo current, these limits were extended, particularly in higher pressures.

It was then clear how Mr. Langden, always working at pressures below $0.1 \mathrm{~mm}$., had always obtained uniform platinum films while

\footnotetext{
${ }^{1}$ Electrical Resistance of Thin Films Deposited by Kathode Discharge, A. C. Lang-
} den, Physical Review, Vol. XI., July-August, igoo. 
the pressures I used (usually exceeding o. $1 \mathrm{~mm}$.) had resulted in colors. Also the explanation of the tiny transparent center, surrounded by a black ring, was clear. This was always obtained at pressures under $\mathrm{O} . \mathrm{I} \mathrm{mm}$. and when the glass was close to the cathode; thus, the plate being within the cathode dark space, received scarcely any of the deposit. On the other hand, at pressures under O. I mm., when the distance between cathode and plate was increased, I obtained a heavy, uniform film. However, whenever color was obtained, I found that the cathode dark space had no effect upon my films. When the distance between cathode and plate was small I could get as good rings as for a great distance, though in the first case the plate was far within the cathode dark space and far outside of it in the other case. Also at a pressure of $12 \mathrm{~mm}$., where, using the dynamo, good rings were obtained, there was no light in the receiver except a purple glow about the knob.

As the pressure is increased from $.05 \mathrm{~mm}$. up to $5 \mathrm{~mm}$. the following stages may be traced in the films formed between these limits; at .05 mm., the lowest pressure used, there was a heavy platinum deposit; at $0.1 \mathrm{~mm}$. a uniform deposit, but more under the cathode and less spreading than for $.05 \mathrm{~mm}$. pressure; at $0.2 \mathrm{~mm}$. a slight trace of color was noticed in the uniform deposit, more frequently on the back of the glass plate; at $0.3 \mathrm{~mm}$. colored rings were formed that were practically transparent and this held up to I $\mathrm{mm}$. pressure; at I $\mathrm{mm}$. the appearance was that of colored rings slightly yellowish in tint surrounded by a transparent zone and beyond the zone an even black deposit. At $2 \mathrm{~mm}$. pressure, the rings were very perceptibly smaller, the transparent zone correspondingly contracted and the outer black deposit thinner and more transparent; from $2 \mathrm{~mm}$. upward, the decrease in size of rings and transparent zone continued and the increase in the transparency of the film also continued. At a pressure of $\mathrm{I} 2 \mathrm{~mm}$. the rings were very small and practically transparent with no other trace of deposit. The above account applies to films, when the distance between the cathode and plate is from zero to six millimeters. When the distance is ten millimeters, a similar description applies until $\mathrm{I} \mathrm{mm}$. pressure is reached. At that point, the large rings began to decrease and the transparent zone was small, a greater increase of 
pressure resulted in a uniform black film and no color. This heavy deposit continued up to near $2.5 \mathrm{~mm}$. pressure then grew thinner as the pressure was increased, ultimately disappearing. (I had previously noted that often, after a deposit, there was a heavy black coating on the aluminum base of the receiver; this coating was more noticeable when a small glass plate was used, thereby exposing more of the base to the rays ; at the same time on the glass, colors, only appeared.) It seems then that transparency is characteristic of low pressure, brown color and boundary zones for a moderately low pressure. For moderately high pressure, two cases exist, depending on the distance of the plate from the cathode and resulting in smaller rings and increase of the black deposit for the shorter distance and heavy black deposit, showing no color for the greater distance. Again for very high pressure there is very little deposit, whatever the distance. The lower the pressure and the greater the distance, the more transparent is the film within the range for good ring formation. The changes occurring in a film, as the pressure is increased from .05 to $5 \mathrm{~mm}$. may be summed up thus: an even black deposit gradually gives way to colored rings; shortly before I $\mathrm{mm}$. pressure is reached, the black deposit again appears at the edges of the plate ; with greater increase in pressure, the rings decrease in size and the black deposit creeps in toward the colored area, reaches a maximum and at high pressure disappears. When the distance between cathode and plate is small, the colors begin at a higher pressure than in the case where the distance is great and the black deposit at high pressures is not so heavy, nor does it conceal the rings completely. For all distances, the appearance at I $\mathrm{mm}$. pressure is characteristic and this may be called the critical pressure. A disturbing element in comparing corresponding films at different distances was due to the glow not being strictly confined to the cathode. If it ran up the glass of the cathode or if a discharge occurred at the top of the receiver, the rings were smaller and took a longer time in formation.

I see no possible explanation of this phenomenon on the basis that the even, black deposits are obtained when the plate is just at the edge of the cathode space, for, while it may apply to results obtained at low pressure, it cannot explain why at high pressure and great 
distance an even deposit is obtained. Again, as pressure increases and the glow decreases, the plate nearest the cathode would ultimately touch the edge of the cathode dark space and receive the even deposit which is contrary to the results obtained. It is a curious fact that, for a distance of $10 \mathrm{~mm}$. an even film may be obtained either at $.2 \mathrm{~mm}$. pressure or at a pressure ten times as great.

In comparing those films made by using the Wehnelt interrupter with those made by the current from the two thousand volt dynamo, it is interesting to note that the two classes are really one and the same; for a given pressure and distance, the appearance of the resulting film is the same in either case.

The attempt was made to arrive at the same stage with each film; let us say the starting of the second set of rings. This could be observed from outside the receiver when the light was good. One could see the brown spot form under the cathode, could see it turning to red, then to blue and then to brownish red again, thus indicating the starting of the second set of rings. Under these circumstances, it was possible to make comparison of the time taken at the different pressures to reach this stage. Although one must keep in mind the fact that an increase in distance between cathode and plate means an increase in time, since the size of the rings is correspondingly increased, nevertheless one can say that in any set of films where the distance is unchanged the time taken to reach the given stage of film formation is the same from the lowest pressure giving rings up to the pressure where the even deposit starts to decrease. Beyond this pressure, the time is increased. Suppose the time of formation between $0.3 \mathrm{~mm}$. and $2 \mathrm{~mm}$. pressure is ten minutes, the time taken to reach the same stage in the rings from $2-5 \mathrm{~mm}$. pressure is almost twenty minutes and beyond $5 \mathrm{~mm}$. pressure, the time is enormously increased, two hours and a half being necessary for a film at $\mathrm{I} 2 \mathrm{~mm}$. pressure. In this connection, it should be stated that films made with the interrupter took a longer time than exactly similar ones made by the current from the dynamo.

To be able to measure the current flowing through my receiver and the fall of potential between anode and cathode, at any chosen pressure, I put an ammeter reading to .OOI ampere in the main cir- 
[VoL. XXI.

cuit and a static quadrant electrometer between anode and cathode. Beginning with the highest pressure used and letting the discharge pass through the receiver, I read the current and voltage for that pressure; taking a stroke of the pump, I again read the three corresponding values. In this way I proceeded by steps to the lowest pressure used in the work. The discharge passed continuously between cathode and plate during this experiment, the accompanying curves give the relation between pressure and voltage and pressure and current respectively and show that down to a pressure of about I $\mathrm{mm}$. the current and also the voltage is practically constant; between I $\mathrm{mm}$. and the lowest pressure used, a slight decrease in

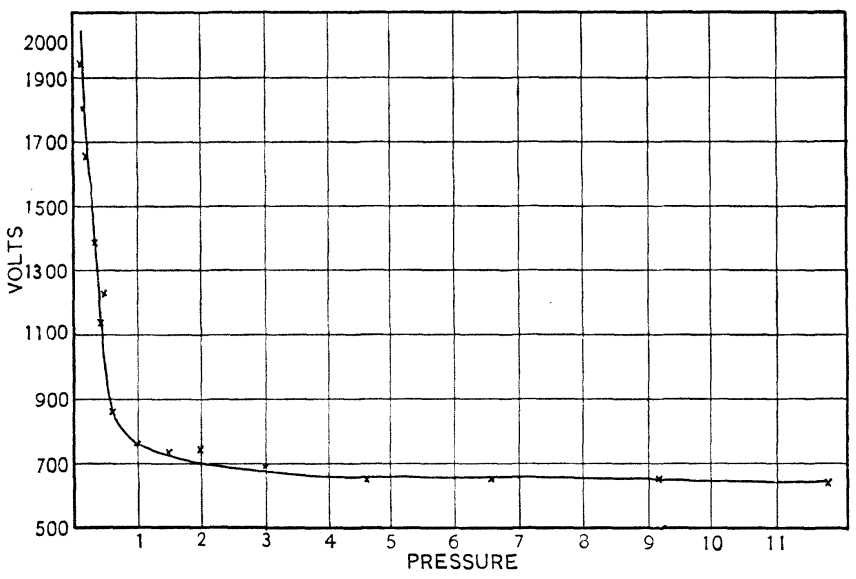

Fig. 3.

pressure is accompanied by great increase in potential and corresponding decrease in current. At four separate times such a series of values were taken and curves constructed (Figs. 3 and 4). In two trials the voltage was constant at about 650 volts and the current constant at .005 ampere until a pressure of $\mathrm{I} \mathrm{mm}$. was reached; then the current fell steadily to .0OI ampere and the voltage rose to I,950 volts; in a third trial, starting with .004 ampere and 600 volts, both factors remained constant until the pressure was decreased to I mm.; between I and $0.5 \mathrm{~mm}$. pressure the change was slight, but below $0.5 \mathrm{~mm}$. the current fell to $.00 \mathrm{I}$ ampere and potential rose to 1,750 volts ; a fourth trial started with a current of .OO I ampere 
and voltage of 550 , both remained constant until $0.5 \mathrm{~mm}$. pressure was reached; then came the sudden drop of current to .000 I ampere and rise of potential to 1,730 volts. Evidently the critical point in pressure for these platinum films is lowered by using a lower voltage and current. If the object is not the lowering of the critical pressure, it is of course far better to use a stronger current and shorten the time of deposit. The current usually used in depositing these films was .004 ampere. In comparing the films by reference to these curves, one finds that the uniform films at pressures below $0.1 \mathrm{~mm}$. are formed when the curve is practically ver-

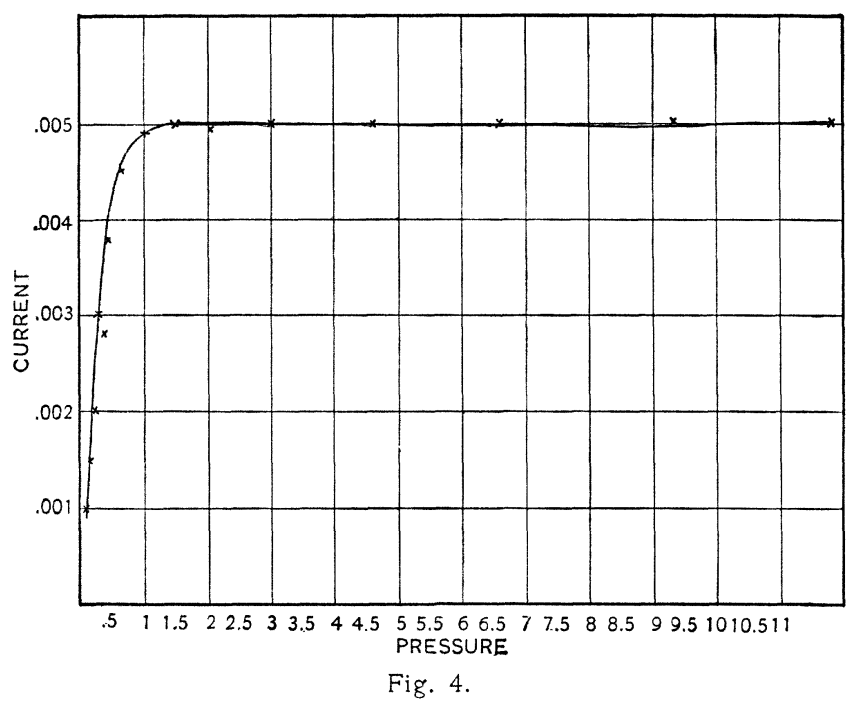

tical; that the colored rings showing greatest transparency and free from all uniform deposits are made when the curve shows a rapid decrease of voltage and rise of current and that the deposit is heavier for those films made at pressures above $\mathrm{I} \mathrm{mm}$. where the curve exhibits itself as a horizontal line. Beyond $3 \mathrm{~mm}$. the decrease in deposit is due to increase in pressure as indicated by the slight glow about the cathode. From observing the curves, I could understand why in the region of pressures below I mm., there would be great fluctuations of voltage, for the small increase in pressure due to gases coming off the cathode at the time of dis- 
charge. Also if a discharge took place at the platinum hook on which the cathode was suspended, the voltage would drop and the current rise, and if, because of the coating with platinum of the glass about the cathode, the glow ran up the glass, again a change would occur. In connection with these curves it is interesting to note that the test plate used in such an experiment became covered with very large rings showing six or seven alternations of color and the deposit of considerable thickness, thus increase of time of deposit would result in the formation of many rings spreading over the surface of the plate.

In order to prove that the colors would form when a gas other than air was used, I arranged to make the deposit in hydrogen. In the first set of films made by the interrupter, I introduced into the receiver hydrogen generated by the action of acid on zinc and dried by passing it through sulphuric acid and phosphorus pentoxide. I pumped this gas through the receiver three or four times before the discharge passed and thus I made a half dozen plates at varying pressures, in each case rings were formed just as in the case of air. To make sure that I had gotten rid of the gases occluded by the cathode and on the walls of the receiver, I resolved to let the discharge using current from the dynamo pass for some time until the pressure of the hydrogen was stationary as indicated by the gauge. Since, with the preliminary discharge, the glass surrounding the cathode would become coated with platinum and in consequence the glow would bend up about the coated glass, it was necessary to protect the glass during the preliminary discharge. Two pieces of mica were taken and bent so as to surround the cathode above and below, shielding the glass about the cathode and the glass plate. Each of these pieces of mica being wound externally with iron wire was removed when the time came by a magnet outside the receiver. These covers having been fitted around the cathode and the receiver made air tight and connected with the pump, the air was exhausted to a low pressure, C. P. hydrogen was admitted and the pressure again reduced. In this way, hydrogen was admitted three times; then the discharge was allowed to pass for two hours. This process was again repeated and during the second discharge, the gauge was watched. At the end of two hours, when the gauge showed no increase of pressure, 
the mica covers were removed from without and the discharge allowed to pass onto the glass plate for twenty minutes, the pressure being $\mathrm{I} \mathrm{mm}$. The rings were like those obtained at the same pressure in air.

I had made attempts to find the thickness of the films by the micrometer calipers, the measurements, however, were of no value even for thick films because of the uneven thickness of the glass and for thin films I obtained negative values.

I then tried weighing a microscope cover glass, putting on a thin film, reweighing and calculating the average thickness of the film from weight, average diameter of circles and density of platinum. By this method, I found the average thickness equal to .000I 36 $\mathrm{mm}$. or the thickness of Newton's first bright ring (.000 I42) approxmately. As this was an attempt to discover how the platinum was deposited on the plate and as I had found that direct observation under the microscope gave me interesting results, I abandoned the weighing method and examined the films one by one under a microscope. When traces of particles were found, the plates were prepared with Canada balsam and cover glasses for use with a high power oil immersion objective. The microscope was then put in front of a lantern and the magnified image obtained on a screen. By running the plate horizontally and vertically, it was possible to examine the whole area and photograph any part of it. Some photographs were taken with objects magnified to 2,000 diameters, others to 2,500 diameters. A film made at a pressure of $.2 \mathrm{~mm}$. showed a field of perfectly spherical particles varying in diameter from .0005 to $.0009 \mathrm{~mm}$. Apparently there was no symmetrical grouping of particles; some were alone, others in large or small groups and here and there an area quite free from them. Each globule was surrounded by a halo. In other films I found many clusters in various stages of development, several globules collected in a faint halo-like area; again a union of many into a rather irregular crystalline appearing area and finally the merging of masses of these crystalline areas into a large solid appearing mass, surrounded frequently by a brown fringe; the photographs illustrate these stages. The thick films shut off most of the light from the lantern and were uniform except where a tear showed the existence of a continuous yellow layer or here and there a crystal- 
line area appeared. Since many of the thin films appeared transparent and the thick ones showed an even yellow deposit, my photographs, for the most part, represented those films where the particles had not yet clustered together to form a continuous film or were taken on the outskirts of the colored area, where the stages of formation could best be observed. Frequently crystalline areas appeared on a uniform field, indicating that particles had been thrown off irregularly after the film proper had formed.

The following explanation seems to fit the observed facts : during the first few minutes of the discharge the platinum thrown off from the cathode is in the form of globules, separated from each other or slightly grouped, hence the deposit appears red; as the discharge continues more globules are deposited, there is a joining together of the groups and the deposit appears blue, edged with red. This, then, would explain the formation of the first set of rings ; the center made up of particles close together, the outskirts containing only separate particles. This process is repeated, the thin, transparent film serving as a base for deposit ; again particles increase directly under the cathode, first, separated from one another and we have red, then uniting together and we have blue; thus a second set of rings have been formed. As this continues, the rings spread out over the surface of the plate and it is evident why extremely thin films and the outer boundary of the colored area, alone exhibit the separate particles.

The particles may be of different sizes; the change in color depends not on the size, but on the grouping of the particles.

It is my intention to examine films showing the first red color and compare with others in which blue first appears; to investigate farther into the formation of even films at great distance and high pressure and to examine the phenomena produced by a cathode of material different from platinum.

I wish the opportunity to express my gratitude to Dr. Hallock for making this investigation possible for me at Columbia University and for his great liberality and kindness to me throughout the accomplishment of the work. I am also greatly indebted to Dr. Davis, Dr. Tufts, Dr. Pegram and Dr. Forbes for valuable advice and for assistance many times.

VASSAR COLlege. 
PLATE 1.

(To face paze 40.)
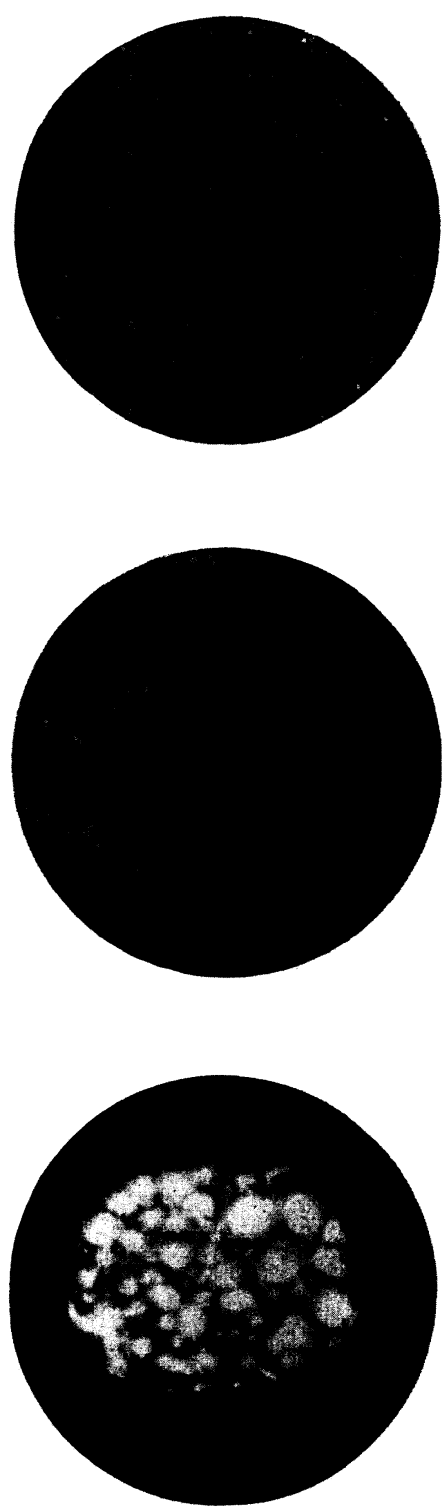

Color in Platinum Films. STONE. 
PLATE

(To face pase 40.)
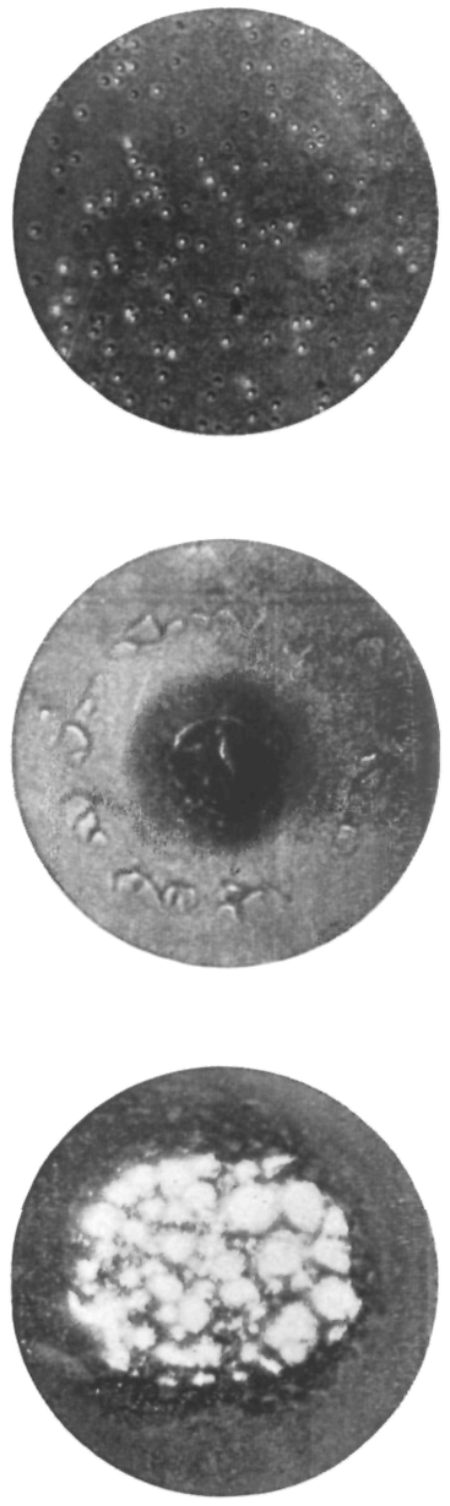

Color in Platinum Films. STONE. 